\title{
EAl Endorsed Transactions

\section{Simulation and Security Calibration of Weather Management System for the Least Rainy Areas of Pakistan through Quantum Key Distribution}

\author{
Nimra Dilawar ${ }^{1}$, Sara Nadeem ${ }^{1}$, Sania $\operatorname{Arooj}^{1}$, Muhammad Rizwan ${ }^{1}$ and Fahad Ahmad ${ }^{1, *}$ \\ ${ }^{1}$ Kinnaird College for Women, Lahore, Punjab, Pakistan.
}

\begin{abstract}
Water plays a vital role for the survival of life. We get water in different forms of precipitation and rain is the most beneficial of all types. The demand for water is very high throughout the Pakistan, especially where we have limited natural water resources. Summer monsoon season has profound impact in reducing water scarcity in Pakistan. Heavy rainfall results into floods whereas less rainfall creates drought which not only affects the economy but also puts human life in risk. The aim of this research is to manage the movement of clouds from heavy rainfall areas to those areas where there is paucity of water, through satellite using electromagnetic waves. To accommodate such a sensitive satellite data which is paramount to a country, it is required to have some special systems based on quantum mechanics that are more efficient and pregnable than conventional computers. The consequences will be very high of not securing weather management system, implies to country level disasters. In this paper we first discuss the technology behind quantum computer then proposed a secure architecture by employing quantum computers to ensure the security of data transmission for weather management system.
\end{abstract}

Keywords: Big Data, Security Calibration, Quantum Computing, Cloud Movements

Received on 26 May 2019, accepted on 30 June 2019, published on 05 August 2019

Copyright (C) 2019 Nimra Dilawar et al., licensed to EAI. This is an open access article distributed under the terms of the Creative Commons Attribution licence (http://creativecommons.org/licenses/by/3.0/), which permits unlimited use, distribution and reproduction in any medium so long as the original work is properly cited.

doi: 10.4108/eai.13-7-2018.159794

*Fahad Ahmad. Email:fahad.ahmad@kinnaird.edu.pk

\section{Introduction}

Water is crucial for our life and we can't survive without it. If there is no water in the soil, then nothing growing on it and everything that depends on it would be dead or lifeless. In 2025, Pakistan is running out of freshwater and authorities anticipate that it would be inadequacy of 31 million acre-feet (MAF) [1]. About 62 percent population of Pakistan is directly or indirectly associated with agriculture, so the deficit will be ruinous for a land whose economy based on agriculture [2].

In Pakistan, summer monsoon season is very important for agricultural, economic and social purposes. These rains are used in agricultural sector to grow healthy and lushly plants. By deluging the fields, certain insects that attacking the field like crickets, white ants can be drowned in rain water and thus crops can be saved. In Pakistan, monsoon contributes almost 60 to 75 percent of the total yearly rainfall [3]. Generally, monsoon is not less than a blessing in the state as it reduces the water shortage and provides enough water to the dams which is used to generate electricity and it also improves power shortage crisis in Pakistan. Further, water management system is more beneficent to manage the water in agricultural, industrial and flooded and rainy areas.

The demand for water is very high throughout the Pakistan especially to deal with drought like situation in Baluchistan and some areas of Sindh [4]. It is vital that rains come in time otherwise delayed monsoon may cause drought. Pakistan is contributing more to aggravate the drought conditions within the country as a third waterscarce country but Pakistan have ability to overcome with drought issue it only requires proper vision and strategy 
[5]. The large amount of data which is large enough to handle through limited processing capabilities and storage through conventional computers is known as big data, so, managing this data is a very big challenge. To deal with big data related to weather which is paramount to a country, quantum computers are used.

Nowadays, efficient calculation cannot be performed in limited time using classical approach like prime factorization of the large integer in a polynomial time. A new pregnable approach based on the laws of quantum mechanics is required to perform such processing on big data using quantum computers. The idea of involvement of the quantum computers in weather management system is to make it computationally more efficient and pregnable. These weather management system needs to be secured and managed in such a way that no one takes over the system. Hence, in this research Quantum based approach is used to secure and analyse the big data for weather management system of Pakistan [6].

\section{Quantum Computers- An Overview}

Quantum Computers is a new trend in the field of computational sciences. It is completely a new paradigm shift from silicon and semiconductor hardware to computers based on laws of Quantum mechanics. Computer systems are becoming smaller and smaller from mechanical computer to vacuum tubes, from transistors to IC's, which has become difficult for classical theory to explain. Thus need of time to move on Quantum theory. The Quantum computers use mechanical phenomena for data processing at atomic and subatomic level. Google and NASA are the pioneers for this paradigm shift as the first Quantum computer has been designed by them manufactured by D-Wave Systems which is installed at NASA in California.

Moore's law stated that after every 18 months, the speed of processor doubles and its size halves which depict that for the processing speed we need have to wait for century. We are blessed that Paul Bernioff at Argonne National Laboratory who formulated the quantum computers in 1981 for a Turing Machine where bits are stored as Qubits, the superimposition phenomena of quantum physics. Each memory location can have values 0 , and both 0 and 1 at a same time [7]. This parallelism property of Quantum computing results into fast and efficient computations. Hence, the power of Quantum computers promises to resolve the hardest problems in computational field i.e., factorization of huge numbers, database search difficulties, discrete logarithms which are not computable through classical computers [8, 9]. Quantum Mechanical theories are totally different from the point of common sense. But it agrees fully with experimental facts, this is the beauty of Quantum Mechanics due to its different properties [10] shows in Table 1.

Table 1. Properties of Quantum Computers

\section{PROPERTIES DESCRIPTION}



2. Uncertainty Principle

Quantum systems are tiny in size and its properties cannot measure until it states are disturbed. Hence, the properties of Quantum system with precision are not possible.

3. Entanglement It is the property states that two particles can be linked when passed through a slit and their properties are linked. If the property of one particle is changed, it affects the properties of other particle without interaction.

4. De-coherence It is the property which states that if a particle interacts with environment will lose its superposed state and change its state from quantum state to classical.

\section{Literature Review}

Cloud in the air is a huge concentration of water vapor when air becomes very cold then water vapor gets converted into small water droplets and those water droplets fall on the ground as rain. The silver iodide or some other substance is used to disperse over cloudy atmosphere. It modifies the weather and it changes the amount of precipitation that falls which helps in condensation process to create large enough water droplets and causes rain fall. This process is known as cloud seeding [11] initiated in 1946 at the General Electric Laboratory in New York [12]. In theory, charged particles are attached to the water droplets' nuclei which enables them to survive for enough time to grow in atmosphere. So, to boost rainfall Mateo system used ionization rather than chemicals.

An electromagnetic distribution method is tested out in Pakistan in which small amounts of electromagnetic energy are used to adjust the path of clouds and moving the clouds from heavy rainfall areas to those areas where rain is most needed, this phenomenon is called "electromagnetic shifting". This method is used to cause artificial rainfall in the Gwadar and Makran areas of Baluchistan [13].

An Outlier detection is a method is surveyed that aims to find objects that have abnormal and inconsistent 
patterns in an input database. This detection can also be helpful in determining the cancerous cells of our brain on early stage. It can be utilized to detect suspicious transactions in different transactions. There are various outlier detection methods. Basically, outlier detection techniques are considered a mapping function. If a point is given in any data set, using its mapping function corresponding outliers will be created. The outlier method been used depending on the characteristics of data set. Previously the outliers deal with static data set, using techniques which are categorized as methods based on statistics, distance, density and clusters. Todays used outliers' methods work on vector based data set using outliers named point, collective, vector, sequence, trajectory and graph [14].

Privacy preserving of large-scale rating data comprises of sensitive and non-sensitive issues. The privacy of personal information of surveyed candidates is compromised on the internet without their permit. A greedy anonymized privacy model has been discussed for the protection of sensitive, large scale rating data for both experimental and practical usage [15].

Technology has been explored day by day with the exponential growth of resources through internet. Trends of gathering accurate, refined and the relevant information through information gathering systems has been increased as the accuracy and integrity of information is the first concern for users arises challenges for choosing the relative information gathering systems. To conquer these challenges, concept-based techniques had been discussed for information gathering systems. Most of the information gathering systems' used techniques follow the semantic concepts-based extraction of research over the web where the semantic representation is different for each system. Lexicon-based representation uses the concept with vocabularies defined in terminological ontologies, thesauruses, or dictionaries, results into overloaded information. Subject-based representation which is most of the time depends on the library systems a limitation for this. Pattern-based representation uses phrases with drawback of phrase length. Semantic concepts based extraction uses different classifier models based on machine learning concepts [16].

The future era is an era of control models having the consistent decision making and technology adaptability capabilities. Some of the biggest challenges for such models are to modelized their constraints such as authorizations, obligations, discussed. An object constraint language (OCL) has been used for the UCON model, also examples discussed to experience its adaptability and flexibility [17].

\subsection{Types of Clouds}

This Clouds in troposphere are divided into majorly three different categories high, mid and low [18] shows in Table 2. Clouds detection is the most challenging task for

weather meteorologists. Different cloud detection techniques have been discussed in the following.

A Semi-supervised Cloud Classification [19] technique used support vector machine classifier based on the combination of clustering and mean map kernel. In this method, the similar cloud images are classified into the same class combining the same class and cluster similarities and combine the labeled and unlabeled pixels to increase the clouds detection efficiency and reliability. This technique is easy to implement but require large training data set.

A neural network approach uses a three layered architecture based on neural network, which compare the $\mathrm{K}$ - nearest neighbor approach and linear discriminant analysis method to classify different types of clouds [20].

\section{Table 2. Types of Clouds}

\section{TYPES DESCRIPTION}

\section{HIGH:}

These clouds' boundaries are approximately 6200 meters high from sea level. Usually they are made of ice crystals.

\section{MID:}

These clouds' boundaries are approximately 2000 to 6200 meters high from sea level. They are made of combination of water droplets with ice crystals.
Cirrus: These are slender, wispy clouds spread over the sky in high breezes. A couple of cirrus clouds not affect the weather, but more clouds demonstrate variation in climate, which happen within 24 hours.

Cirrocumulus: These clouds are called the sky of the mackerel. When cirrus clouds change into cirrocumulus, tropics result in tempests, results in typhoon.

Cirrostratus: These clouds are tiny sheets that spread across the sky. The sky shading ends up yellowish, white, and translucent. For the most part, these clouds appear one or half day before a rainstorm.

Altocumulus: These clouds made up as layers of dull, round, little water droplets' clouds. Altocumulus clouds turn into rainstorms late into the evening on a sunny hot weather morning. 


\section{Cumulus:}

These clouds are known as goodweather clouds as they tend to show good, dry weather. It's bright when there are chances of rain. With rounded piles or scoops on top, the water droplets' clouds have a flat base. They are called cumulus congestus or elevated cumulus when the scoops look like cauliflower heads. They are able to be at a very height.

Cumulonimbus: These clouds are clouds of thunder that have developed from clouds of cumulus. Most of their edges are very dim. These clouds can predict severe weather, such as torrential rain, hail, snow, storms, tempests and whirl wind.

The clouds' boundaries are altitudes beneath 2000 meters. They are made of water droplets.

Stratus: These clouds are dull grayish clouds extending over the sky and blocking it. They look like heavenly fog. Also known as overcast is the Stratus spread. They become haze when their boundaries touch the ground. They can make quality snow or shower.

Stratocumulus: These clouds are low, puffy and dim. They show dry climate if there are slight contrasts in temperature among night and day. Precipitation is uncommon, yet it can transform into clouds of nimbostratus.

Nimbostratus: The dim, wet-looking shady layer of these clouds is thick to the point that it hinders the Sun totally. They regularly produce downpour with and without snow precipitation. Precipitation can keep going for quite a while.

Cloud Detection and Removal Algorithm for MODIS Remote Sensing Imager is discussed in [21]. Moderate Resolution Imaging Spectrum-radiometer (MODIS) is onboard earth observing system used to detect and remove clouds. This technique is useful to identify cloud and ground area based on the analysis of multispectral characters derived from MODIS imagery. This approach is useful but takes more time to process with high misclassification rate.

Cloud-Screening Algorithm [22] is proposed comprises a general system for multispectral images obtained through radars in orbit around the earth, in the detectable and near-infrared spectral range which specifies the atmospheric-oxygen and water-vapor absorptions. This approach has improved the classification technique, but it also takes time.

The two core categories are Cryptographic and NonCryptographic. The cryptographic organisations hire encryption methods, specifically: symmetric key encryption, public key encryption and numerous cryptographic primitives, however non-cryptographic methods embrace access control techniques such as RBAC, ABAC, IBAC etc. [23].

In various situations, data benefactors have not any/slight control that has directed to fear that the private data may be stranded. Microaggregation methods hunt for shield microdata in such a way that data can be available and extracted without providing any secret evidence that can be connected to definite people [24].

The delinquent with entree regulator approaches to impose discriminatory entree to outsourced data without linking the possessor in endorsement is addressed here. The elementary impression is to associate cryptography with endorsements, and data possessors allocate keys to persons that will impose entree through encryption. A recognized entree model is intended to examine the rendering an endorsement strategy into an corresponding encryption strategy [25].

\section{Objective}

The objective of this research is to engage quantum computing-based approach for the movement of clouds from the heavy rainfall areas to least rainy areas to analyze the big data of satellites for weather management system of Pakistan.

\section{Components of Proposed Model}

Constantly Taking Pictures Satellite (CTPS), Satellites' Signals Capturing System (SSCS), Quantum Computers, at Clouds' Movement Managing Satellite (CMMS) and Quantum key distributor (QKD) are the key components of proposed architecture.

\subsection{Constantly Taking Pictures Satellite}

The quantum computing based Constantly Taking Pictures Satellite (CTPS) is launched in space to capture the images data of current weather which is securely downlink towards ground station after encryption through Quantum key distributor.

\subsection{Ground Station}

The quantum computing based Constantly Taking Pictures Satellite (CTPS) is launched in space to capture the images data of current weather which is securely downlink towards ground station after encryption through Quantum key distributor. 


\subsection{Satellites' Signals Capturing System}

This The encrypted data of CTPS is received at the ground station for further processing by Quantum Computers through Satellites' Signals Capturing System (SSCS) composed of

- Antenna and its tracking system

- Transceivers, LNA and accessories for interfacing antenna with control Quantum computer

- Control Quantum computer connected to remote servers through intranet.

\subsection{Quantum Computers}

This Quantum Computers are implanted at the ground to decrypt and processed the encrypted images of weather through SSCS, to extract the required information of clouds to be moved from one location to another. The processed information is again encrypted and sends towards another SSCS to forward towards CMMS, another satellite launched for actual movement of clouds.

\subsection{Clouds' Movement Managing Satellite}

The encrypted information of Quantum Computers is received at Clouds' Movement Managing Satellite (CMMS) in the form of signals which decrypt the information through Quantum Key Distributor to generate signals to move clouds from heavy rain fall areas to least rainy areas.

\subsection{Quantum Key Distributor}

The Quantum key distributor (QKD) has been implanted in each component of proposed model described above using BB84 protocol. QKD is a highly secured cryptographic protocol based on quantum computing. QKD technique encrypts or decrypts data using two shared channels for key transmission and data transmission respectively. The key shared channel is a quantum channel where a shared key travel from sender to receiver. The data is transmitted over a classical channel after encrypted with the key. This feature of QKD makes it secure and detects the presence of eavesdropper, trying to capture the key unethically.

The key is transferred on the quantum channel using single photon, precisely decoy state of single photon. The purpose of using single photon is that the eavesdropper could not create the clone of transmitted photon. If the eavesdropper tries to capture the data, then the photon will lose its superposed state.

\section{BB84 Protocol}

QKD has been implanted for BB84 protocol utilizing photon polarization states to transmit the data where any two sets of conjugate states can be selected. Alice as sender and Bob as receiver are associated by a quantum communication channel which permits Quantum states to be transmitted Figure-1. The channel used can be a fiber optic channel or free space. Other channels, for instance utilizing communicate radio or the web. The protocol is structured with the suspicion that a stranger (Eavesdropper) can meddle in any capacity with the quantum channel, while the established channel should be verified.
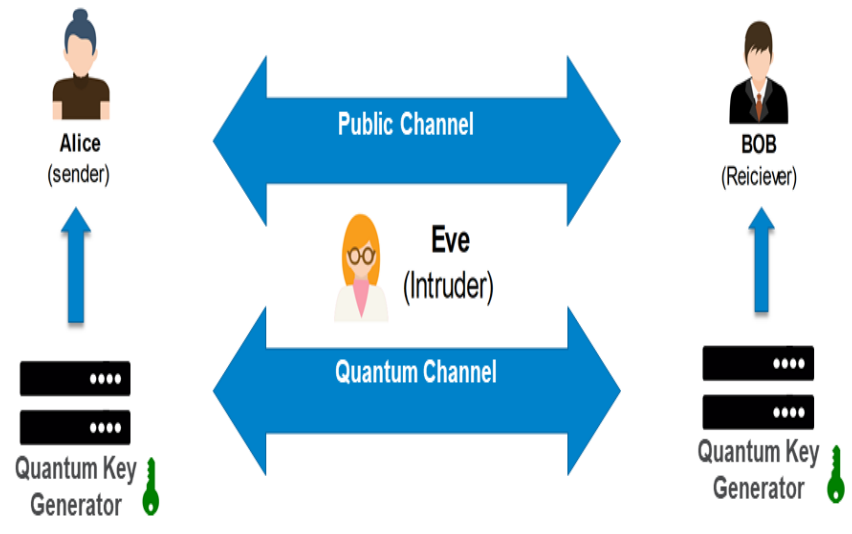

Figure 1. Quantum Key Distribution Method

The security of the protocol made sure using encrypted data on orthogonal basis. Quantum's approach states that these states can't be estimated until the states are disturbed, due to superposition state. BB84 uses two sets of states, where each set is conjugate to the next one, and the two states inside a set are perpendicular to one another. The standard polarization sets of orthogonal states, basis utilized, and either the straight basis of vertical at 0 degree and horizontal as 90 degree, the 45 degree and 135 degree as diagonal basis. Any two of these bases are conjugate to one another, thus any two can be used in the protocol. Underneath the rectilinear and diagonal bases are utilized.

\section{Proposed Model}

The main flow of proposed model has been described as a Constantly Taking Pictures Satellite (CTPS) captures images of clouds from space within in the interested territory continuously. The captured data is gathered for secured transfer through QKD method. After encryption, CTPS downlink the data packets containing images towards ground stations through classical channels.

At the ground station, Satellites' Signals Capturing System (SSCS) is implanted to receive the encrypted data which is forwarded towards a Quantum Computer for processing. Quantum Computer decrypts the received data packets through QKD. The decrypted required information is extracted, or the data is processed about navigation of clouds from heavy rainfall areas to the least rainy areas. The processed information is forwarded after again encryption through QKD to another SSCS, which uplinks 
the channel for the transmission of data signals towards another satellite Clouds' Movement Managing Satellite (CMMS), responsible for managing the clouds movement. The encrypted information is decrypted hereabout the sensitive information of clouds movement regions and accordingly signals are generated to move clouds from heavy rainfall areas to low rainfall areas through QKD method. Ground stations use the transceivers which provide interface between ground station and quantum computer also known as the control Quantum computer.

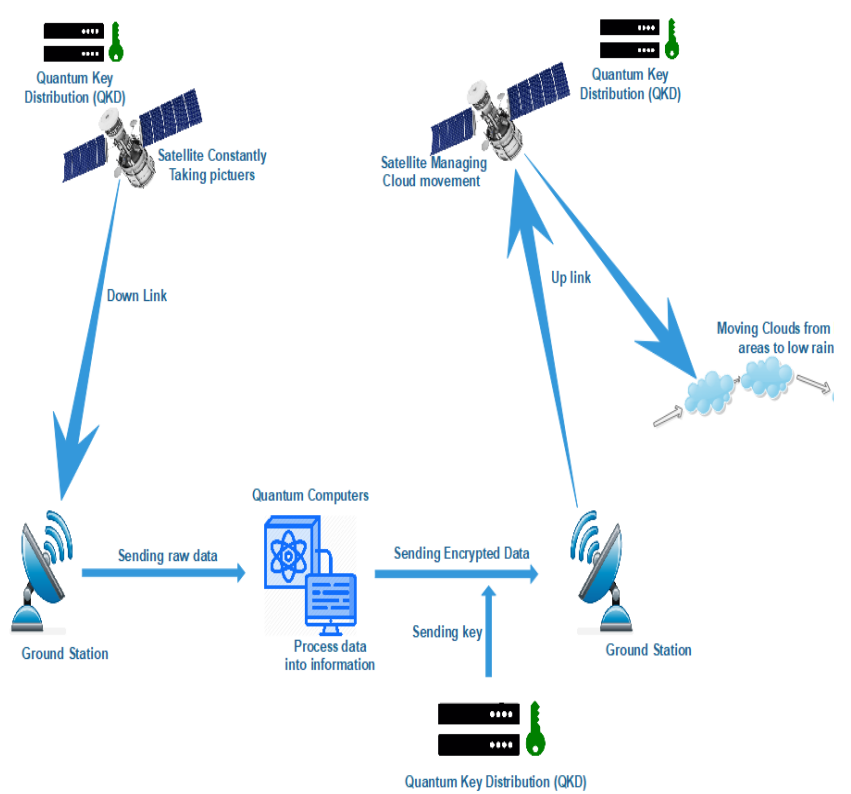

Figure 2. Proposed Architecture

\section{Theory behind Technique}

Image processing and segmentation identification plays a vital role in the extraction and interpretation of information from images obtained from satellites. Constantly Taking Pictures Satellite (CTPS) provides an opportunity to monitor the weather's data continuously in real time at a high spatial resolution, using visible imagery.

The main issue with the gathered images is that they haven't clear boundaries and edged. The cloud image ad its boundaries are almost smudged which makes segmentation, a difficult process.

The other issue is that the shapes of clouds although not look like to be changed with seconds but for the image processing, each pixel changed its position rapidly and each image segment vary with every second of time.

However, different clouds exist at different levels of height in the atmosphere. Hence, image processing makes it possible to identify each cloud segment in different time.

\section{Difference Centroid Algorithm}

The required cloud segments are calculated from each image of the CTPS, the distance of the cloud's movement is calculated through the distance of their centers.
Suppose the centroid of the cloud of the reference image taken at time $\mathrm{t}_{\mathrm{i}}$ is $\left(\left|\psi_{x i}\right\rangle,\left|\psi_{y i}\right\rangle\right)$ and that of the next image at time $\mathrm{t}_{\mathrm{j}}$ is $\left(\left|\psi_{x j}\right\rangle,\left|\psi_{y j}\right\rangle\right)$ then the displacement $\left|\Psi_{D}\right\rangle_{i j}$, or the distance traveled can be deduced as follows.

$$
\mid \Psi_{D}>_{i j}=\sqrt{\left(a\left|\psi x_{i}>-b\right| \psi x_{j}>\right)^{2}+\left(c\left|\psi y_{i}>-d\right| \psi y_{j}>\right)^{2}}
$$

The centroid of the cloud segment is defined as

$$
\left|\psi_{x}>=\frac{\sum \Psi_{x i}}{\psi_{N}} \quad\right| \psi_{y}>=\frac{\sum \psi_{y i}}{\psi_{N}}
$$

Where $\mathrm{S}$ is the cloud segment and $\left(\psi_{x^{\prime}} \psi_{y}\right) \varepsilon \mathrm{S}$ and number of pixels are represented by $\mathrm{N}$

The speed $\mathrm{V}_{\mathrm{ij}}$ and direction $\theta$, of the cloud segment is calculated as follows

$$
\left|\psi_{v}>=\frac{\mid \Psi_{D}>_{i j}}{t_{j}-t_{i}} \quad\right| \theta_{i j}>=\tan ^{-1}\left(\frac{\Psi_{y j}-\psi_{y i}}{\Psi_{x j}-\psi_{x i}}\right)
$$

After calculating the velocity and direction of the cloud we can find the angle for the movement of the cloud in a specific direction in a specific area of Pakistan. The only need is to have both positions to where and from where clouds need to be moved.

\section{Simulation}

\section{Case I}

In this scenario, bases are fixed. Eavesdropper choose the same base $\mid \mathrm{V}>$ as Alice (sender) but she fails to capture the session, but due to coherence photon destroyed. So, Bob (receiver) is unable to decrypt the data as shown in Figure-3.

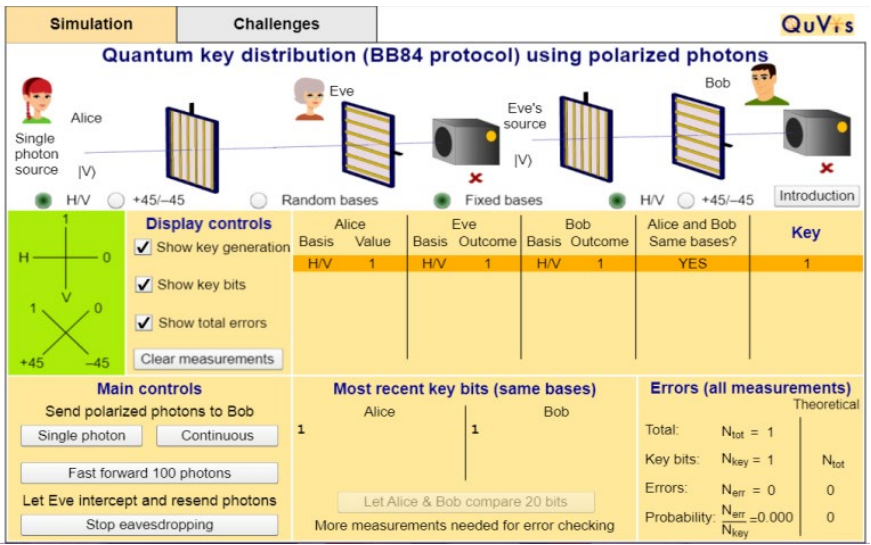

Figure 3. Session not captured but data destroyed 
In this scenario, bases are fixed. Eavesdropper tries to capture the session but failed. Bob (receiver) receives the session successfully. The outcome bit generated against this case is 1 because Bob (receiver) successfully received the photon. Data is decrypted by the bob as shown in Figure-4.

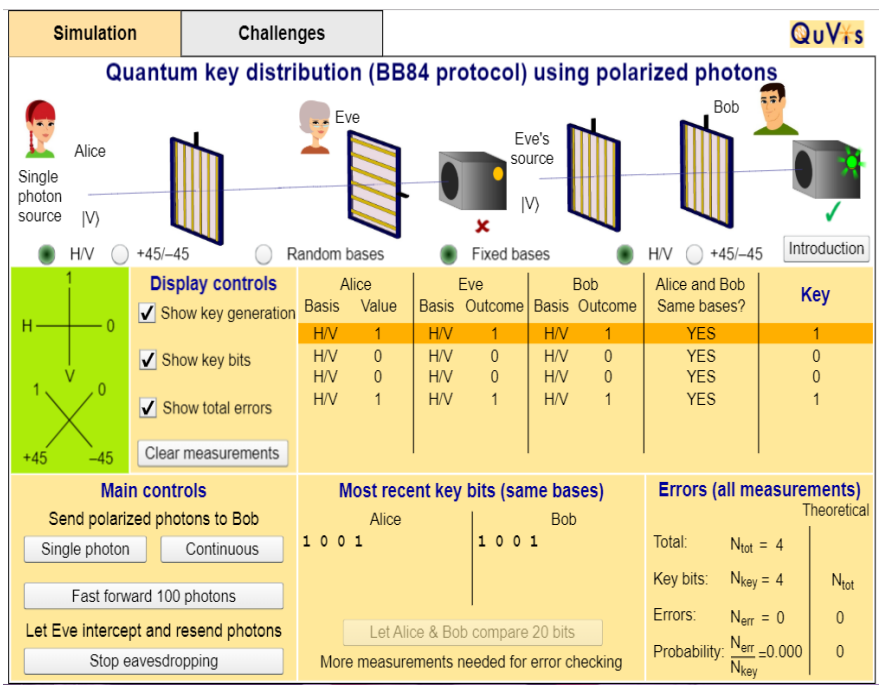

Figure 4. Session not captured and data received
successfully

\section{Case III}

In this scenario, bases are fixed. Eavesdropper tried and captured the session and Bob (receiver) is unable to decrypt the transferred data hence, the key generated in this case is 1 as shown in Figure- 5.

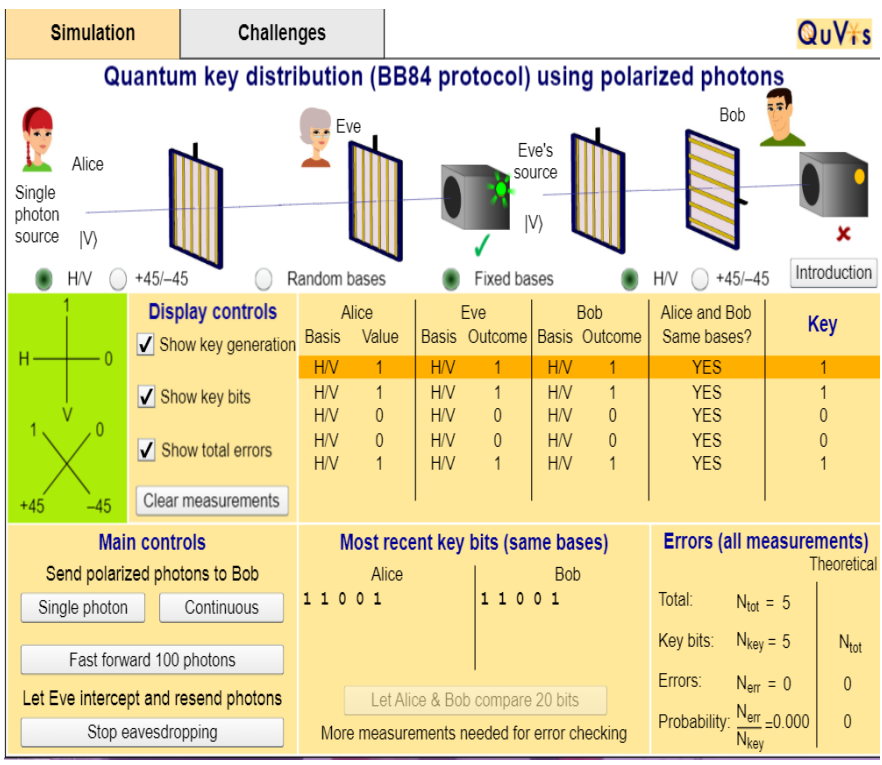

Figure 5. Session captured but data not received

\section{Challenges and Future Research Directions}

Although at this time, quantum systems are seemed to be highly secured and the security of processed data isn't challengeable, but the security of these systems would be the biggest threat for countries in the quantum future world, not only at international level. But also at national level with exacerbate vulnerable challenges in the democratic parliamentary countries where political system based on an elected form of governance is followed. In such countries, a conflict within provinces poses significant security risks and grave challenges to resources occupied regions.

To maximize the resources' usefulness, their even distribution within country and to eradicate the high security threats in the future quantum world, there would be need of implication of strict legislation followed by highly encrypted systems. Hence this paper contributes to the scholarly future debate on the complex relationship between weather management systems and their security.

\section{Conclusion}

The agricultural, economic operations and security of a country depends on the weather conditions. Hence, Quantum Computers with exponential parallel processing capacity managed the movement of clouds and holds the promise for pregnable prosper future of a country. In future we will try to figure out the other uncertain situations means i.e. natural disaster. Moreover, we will propose a methodology which maintains the temperature level to manage the atmosphere for long period of time.

\section{References}

[1] Raza, M.M., Thirsty Days Ahead: Pakistan's Looming Water Crisis, in The Diplomat. 2018.

[2] Khan, W.M., Year Book M.o.F.a. Agriculture, Editor. 2009.

[3] Ahmad, I., et al., Probability Analysis of Monthly Rainfall on Seasonal Monsoon in Pakistan. International Journal of Climatology, 2014. 34(3): p. 827-834.

[4] Syed Shahnawaz Ali, S.K.a.A., Muslimaid Pakistan Drought Situation in Pakistan. 2019.

[5] Sheikh, M.M. Drought management and prevention in Pakistan. in COMSATS 1st meeting on water resources in the south: present scenario and future prospects, Islamabad. 2001.

[6] Seo, J.W. and S.J. Lee, A study on the integrity and authentication of weather observation data using Identity Based Encryption. SpringerPlus, 2016. 5(1): p. 1225.

[7] Bennett, C.H., et al., Strengths and weaknesses of quantum computing. SIAM journal on Computing, 1997. 26(5): p. 1510-1523.

[8] Datta, A., A. Shaji, and C.M. Caves, Quantum discord and the power of one qubit. Physical review letters, 2008. 100(5): p. 050502. 
[9] Ferraro, A., et al., Almost all quantum states have nonclassical correlations. Physical Review A, 2010. 81(5): p. 052318.

[10] Chintan Bhatt, T.S., Quantum Computing: Fusion of Physics and Computers, in Technical Trends. 2015.

[11] Guo, R., Cross-Border Environmental Pollution and Management. 2018: Elsevier

[12] Eckhardt, G. The Edward Aquifer Website. 2018.

[13] Ahsan, A., Artificial Rain in Balochistan: Game Changer or Wishful Thinking, in Propakistani. 2018.

[14] Zhang, J., Advancements of Outlier Detection: A Survey. ICST Transactions on Scalable Information Systems, 2013. 13(1): p. 1-26.

[15] Sun, X. and L. Sun, Privacy Preserving Large-Scale Rating Data Publishing. EAI Endorsed Transactions on Scalable Information Systems, 2013. 13(1-3).

[16] Tao, X., et al., Personalised Information Gathering and Recommender Systems: Techniques and Trends. IEICE Transactions on Information and Systems, 2013. 13(1-3): p. e4.

[17] Li, M. and H. Wang. Specifying Usage Control Model with Object Constraint Language. in 2010 Fourth International Conference on Network and System Security. 2010. IEEE.

[18] Observing Clouds and Weather. 2015; Available from: https://www.sciencelearn.org.nz/resources/628-observingclouds-and-weatsher.

[19] Gómez-Chova, L., et al., Mean map kernel methods for semisupervised cloud classification. IEEE Transactions on Geoscience and Remote Sensing, 2009. 48(1): p. 207-220.

[20] Lee, J., et al., A neural network approach to cloud classification. IEEE Transactions on Geoscience and Remote Sensing, 1990. 28(5): p. 846-855.

[21] Gu, L., R. Ren, and S. Zhang, Automatic Cloud Detection and Removal Algorithm for MODIS Remote Sensing Imagery. JSW, 2011. 6(7): p. 1289-1296.

[22] Gómez-Chova, L., et al., Cloud-Screening Algorithm for ENVISAT/MERIS Multispectral Images. IEEE Transactions on Geoscience and Remote Sensing, 2007. 45(12): p. 4105-4118.

[23] Chenthara, S., et al., Security and Privacy-preserving Challenges of e-Health Solutions in Cloud Computing. IEEE Access, 2019.

[24] Kabir, E., et al., Microaggregation sorting framework for k-anonymity statistical disclosure control in cloud computing. IEEE Transactions on Cloud Computing, 2015.

[25] Wang, H., et al., Protecting outsourced data in cloud computing through access management. Concurrency and computation: Practice and Experience, 2016. 28(3): p. 600615. 